\title{
EL DIMINUTIVO EN LA BOLIVIAANDINA DE LA PRIMERA MITAD DEL SIGLO XIX: VALORES Y FUNCIONES EN EL DIARIO DE J. S. VARGAS
}

\author{
José Luis Ramírez Luengo \\ Universidad Autónoma de Querétaro (México) \\ joseluis.ramirezluengo@gmail.com
}

\begin{abstract}
RESUMEN: El empleo del diminutivo es un fenómeno de primera importancia para la caracterización del español andino dentro de las variedades americanas de esta lengua, no solo por su altísima frecuencia de uso, sino también por los numerosos valores semánticos que presenta y por su aplicación a bases morfológicas que no admiten tales elementos en otras variedades del español. El presente trabajo se propone ofrecer una descripción del uso que hace del diminutivo un hablante del occidente de Bolivia durante la primera mitad del siglo XIX, con el propósito de determinar si la situación existente actualmente se daba ya en esta época o es un fenómeno más moderno en la variedad lingüística mencionada.

PALABRAS CLAVE: Historia de la lengua, morfosintaxis, español de Bolivia, siglo XIX, diminutivo, contacto de lenguas.
\end{abstract}

\section{DIMINUTIVE IN ANDEAN BOLIVIA DURING THE FIRST HALF OF 19TH. CENTURY: VALUES AND FUNCTIONS IN J. S. VARGAS' DIARY}

\begin{abstract}
The use of the diminutive is a very important phenomenon for the characterization of Andean Spanish among the American varieties of this language, because of its very frequent utilization, its different semantic values and also its application to morphological basis which don't accept these elements in other Spanish dialects. This paper aims to offer a description of the use of the diminutive that an Andean Bolivian speaker makes during the first part of $19^{\text {th }}$ century, in order to establish if the modern situation concerning these morphemes already exists in this moment or it is a recent phenomenon in Bolivian Spanish.

KEYWORDS: History of the Spanish Language, morphosyntax, Bolivian Spanish, 19th. Century, diminutive, languages in contact.
\end{abstract}

Recibido: 24/02/2016. Aceptado: 08/07/2016 
1. Como es de sobra conocido, el empleo del diminutivo constituye uno de los fenómenos que de manera más clara identifican y caracterizan al español andino dentro de las variedades americanas de esta lengua, no solo por su altísima frecuencia de uso, sino también por los numerosos valores semánticos que presenta y, como consecuencia de lo anterior, por la aplicación a bases que no admiten tales morfemas en otras variedades del español ${ }^{1}$. Así -y en el caso concreto del español del occidente de Bolivia-, todos los autores que se ocupan de su descripción (entre otros, Coello Vila, 1996: 177; Mendoza Quiroga, 2008: 229) recalcan el uso notablemente más abundante de estos elementos en comparación con el registrado en otras variedades dialectales y, sobre todo, su empleo en categorías gramaticales donde, en general, no resulta tan frecuente o directamente no se acepta, tales como el adverbio, el pronombre o los numerales y posesivos, con ejemplos como esito, biencito, allacito, lueguito (Mendoza Quiroga, 2008: 230) o cuatrito y suyito (NGLE, 2009: 634, 1398)2.

Por lo que se refiere a los orígenes de este uso en determinadas variedades americanas del español, Reynoso Noverón (2001-2: 116) propone una "posible activación por contacto", esto es, que "la proliferación de diminutivos en el español de América y su uso en categorías poco usuales en otras variantes puede deberse a una reactivación, actualización y reajuste de los patrones polisémicos que han caracterizado al diminutivo desde el latín, debido al contacto lingüístico y cultural establecido en América a partir del descubrimiento", idea a la que -en el caso concreto del español andino- se suman autores como Caravedo (1992: 295), Godenzzi (1996: 90) o el ya mencionado Mendoza Quiroga (2008: 229)33.

Ahora bien, conviene señalar que, más allá de la situación que se descubre en la sincronía y de las causas que llevan ella, son muchas las cuestiones que aún

1. Si bien es cierto que esta descripción es válida para todo el español de América -a este respecto, señala muy acertadamente Reynoso Noverón (2001-2: 112) que “su abundante y para algunos 'excesivo' uso en contextos y entidades que muy poco o nada tienen que ver con la disminución, ha sido establecido como una diferenciación reconocida hasta por los propios hablantes entre el español americano y el peninsular"-, también lo es que resulta especialmente apropiada para algunas variedades como el ya mencionado español andino o el del altiplano central mexicano, en contraste con otras como pudiera ser, por ejemplo, el español rioplatense, donde tal uso excesivo no es tan marcado o evidente.

2. Tal estado de cosas da la razón a Lázaro Mora (1999: 4650-1) cuando indica que, más allá del sustantivo y del adjetivo, en realidad prácticamente todas las bases léxicas pueden recibir sufijación apreciativa de tipo diminutivo.

3. Todos estos autores defienden la influencia del quechua -en concreto del sufijo -cha, "que, aparte de indicar tamaño pequeño, puede también denotar afección o desprecio” (Mendoza Quiroga, 2008: 229)-, a la que el profesor paceño suma, para el caso del occidente boliviano, la de la lengua aimara y el sufijo -lla, de idéntica significación (Mendoza Quiroga, 2008: 229). 
se deben responder acerca de este fenómeno, entre las que destaca sin duda todo lo referente a su diacronía, y en concreto al momento en que se impone el estado de cosas que se ha descrito ya para el periodo actual ${ }^{4}$; se hace necesario, por tanto, un estudio histórico que, sobre corpus documentales adecuados, determine la época en que se produce tanto la intensificación en el uso del diminutivo como la ampliación de las bases a las que se aplica, a partir de lo cual se podrán aportar datos que ayuden a responder cuestiones tan relevantes como la relación entre ambos procesos o sus causas últimas -esto es, si se debe al contacto lingüístico con las lenguas autóctonas o no-, y, en definitiva, contribuyan a comprender de manera más profunda lo que sin duda constituye uno de los más importantes marcadores dialectales del español andino en general y de la variedad del occidente boliviano en particular.

2. Con el propósito de responder algunas de las cuestiones planteadas más arriba, el presente trabajo se propone ofrecer una descripción del uso que hace del diminutivo un hablante del occidente de Bolivia durante la primera mitad del siglo XIX, en concreto José Santos Vargas en su conocido Diario (Vargas, 2008) ${ }^{5}$; por supuesto, resulta de rigor señalar que ni el informante ni la época analizada han sido seleccionados al azar, sino que su elección responde a cuestiones de primera importancia en la historia del español de Bolivia, lo que, en consecuencia, determina el interés de un análisis como el que se pretende llevar a cabo en estas líneas.

4. Tales cuestiones han sido estudiadas, por ejemplo, para el caso del altiplano central mexicano, acerca del cual Reynoso Noverón (2001-2: 123) concluye que "justo en el momento en que México alcanza su independencia política y la autodefinición de su identidad es cuando (...) el uso de diminutivos aumenta notablemente convirtiéndose en una característica identificadora del español mexicano”; respecto a sus causas últimas, esta misma autora señala que "esa elevada frecuencia [del diminutivo] está íntimamente ligada a la convivencia entre dos culturas que reflejan su visión del mundo a través de sus respectivas lenguas y al complejo proceso de mestizaje que se llevó a cabo y que parece tener su momento cumbre en el siglo XVIII, con el nacimiento de una nueva cultura y con ello de una lengua mexicana" (Reynoso Noverón, 2001-2: 124). A conclusiones semejantes llega en su magnífico análisis Company (2007), donde relaciona, además, este fenómeno con otros que también se desarrollan en este momento y muestran una pauta de comportamiento semejante.

5. Se trata de una obra en la que el autor describe su vida en una de las guerrillas que, durante la Guerra de Independencia, salpican el territorio andino y preandino de la actual Bolivia, y constituye una fuente de primera magnitud para el conocimiento de la vida cotidiana de los grupos armados partidarios de la emancipación americana durante la larguísima contienda que se vive en la zona altoperuana; esto justifica su empleo en numerosos trabajos históricos que analizan el periodo, entre otros el clásico de Arnade (1972), Machicado et al. (2009) o Mamani Siñani (2010). 
Por lo que se refiere al autor del texto, conviene señalar que José Santos Vargas nace en Oruro en 1796 y, tras enrolarse como tambor patriota en los primeros años de la Guerra de la Independencia, desarrolla toda su vida en la zona de los valles -entre los actuales departamentos de La Paz y Cochabamba-, donde todavía vive al redactar la versión final de su obra ${ }^{6}$; se trata, pues, de un hablante de la variedad de español propia del occidente boliviano que presenta en su escritura, además, muchas características de las manos inhábiles (Marquilhas, 2000: 234-41), lo que sin duda acrecienta la importancia de su Diario como testimonio de la situación de tal variedad en los estratos populares de la primera mitad del siglo XIX ${ }^{7}$. Conviene señalar, además, que, junto al español, Vargas presenta un dominio más o menos alto de las dos grandes lenguas regionales quechua y aimara- (Mendoza, 2008: XXVIII), lo que añade interés al análisis de su empleo de los diminutivos, habida cuenta de que, como se indicó más arriba, en ocasiones se han achacado las particularidades que tales elementos muestran en su uso precisamente al contacto del español con tales lenguas.

En cuanto a la época seleccionada -las primeras cinco décadas del siglo XIX-, se hace necesario mencionar aquí, aunque sea de forma superficial, la trascendencia que presenta tal periodo para la historia del español americano: en efecto, como se señala en Ramírez Luengo (2011: 15-17), las transformaciones políticas que conllevan los procesos emancipadores van a determinar poco a poco la normativización de los usos propios de cada una de las naciones independientes (o, con mucha frecuencia, de sus capitales) como estándar culto regional; al mismo tiempo, este siglo va a asistir a "la consolidación definitiva del español entre prácticamente todos los estratos de las sociedades hispanoamericanas" (Ramírez Luengo, 2007: 28), proceso que determina la hispanización de los hablantes de lenguas autóctonas y que conlleva inevitablemente la aparición de variedades indigenizadas del español, en las que, por tanto, pueden aparecer fenómenos como el que se pretende analizar en estas líneas ${ }^{8}$. Salta a la vista, por tanto, la especial trascendencia que el siglo XIX posee para la definitiva configuración de la variedad que hoy se identifica como propia del

6. Para un estudio histórico minucioso del autor y del texto, es básico consultar el trabajo fundacional de Gunnar Mendoza (2008: XXIII-XLVI), así como el más reciente de Demélas (2007).

7. Sorprende por ello la poca atención que se ha concedido a este texto como corpus para estudios de tipo lingüístico; cabe señalar, con todo, que existen ya algunos trabajos que lo utilizan como base de análisis, entre los que destacan Frago (2011) para cuestiones muy variadas, Gómez Seibane (2012) para los usos pronominales o Gómez González (2013) para el léxico.

8. Dando por buena, por supuesto, la teoría del origen de estos usos a partir del contacto lingüístico entre el español y las lenguas andinas que defienden, entre otros, los ya mencionados Caravedo (1992: 295), Godenzzi (1996: 90) y Mendoza Quiroga (2008: 229). 
occidente de Bolivia y, en concreto, para el estudio de algunas de sus características más prominentes como es la utilización del diminutivo.

Así pues, el trabajo comenzará por ofrecer un listado de los diminutivos que aparecen con tal función en el texto, para después estudiar dos cuestiones fundamentales: 1) el sufijo empleado y determinadas cuestiones relacionadas con su morfología; y 2) los valores semánticos -de tipo referencial o pragmático- que presentan estos elementos y su frecuencia de empleo, siguiendo en esto el análisis de Company (2007). Posteriormente, se terminará por describir la extensión de estos elementos a categorías gramaticales donde no resultan tan habituales y se plantearán algunas cuestiones que se pueden extraer de tal estado de cosas. De este modo, se puede decir que los propósitos del presente trabajo son dos: por un lado -y como se indicó ya en diversas ocasiones-, describir el uso del diminutivo en un hablante bilingüe del occidente de Bolivia de la primera mitad del siglo XIX; por otro, y como consecuencia de lo anterior, establecer si la situación descrita para la sincronía actual existe ya en este periodo o, por el contrario, es el resultado de procesos que van a tener lugar en momentos posteriores ${ }^{9}$.

3. El análisis del corpus ofrece un total de 145 sufijos diminutivos empleados con tal función, esto es, no presentes en voces “opacas, no transparentes o lexicalizadas” (NGLE, 2009: 635) ${ }^{10}$. Aunque resulta difícil valorar su frecuencia de uso por falta de estudios que puedan considerarse bases adecuadas de comparación -sea por la diferencia de criterios aplicados a la hora de determinar tal frecuencia de empleo (Reynoso Noverón, 2001-2: 114-5; Ramírez Luengo, 2006: 41-42), sea por la utilización de tipologías diferentes como base de

9. A estos propósitos -y como consecuencia de ellos- se puede sumar uno más, de carácter mucho más general: aportar nuevos datos que certifiquen la importancia del siglo XIX en la configuración de la situación que ofrece actualmente el español americano (Ramírez Luengo, 2011: 15-9).

10. Como se indica en Ramírez Luengo (2005a: 30-1), “es evidente que, si lo que se pretende es describir el uso del diminutivo en determinado momento, se deberá atender exclusivamente a los sufijos que se emplean con este valor, y dejar aparte aquellos que forman unidades léxicas independientes y que pueden, por tanto, distorsionar sus proporciones reales de utilización”; con todo, es necesario señalar también que los conceptos de ‘transparencia' y 'opacidad' son “nociones graduales, puesto que se basan en la conciencia lingüística del hablante, siempre variable, a menudo dependiente de su cultura particular y, por tanto, difícil de objetivar” (NGLE, 2009: 635-6), lo que añade problemas a la cuestión. En este caso concreto, se ha considerado diminutivo lexicalizado aquel que forma unidades léxicas presentes en el DRAE (2014) y cuya definición no se explica como resultado del proceso de disminución de la base (a manera de ejemplo, guerrilla). 
análisis (Fontanella de Weinberg, 1987: 110; Ramírez Luengo, 2005b) ${ }^{11}-$, lo cierto es que esta cantidad parece contradecir la escasez cuantitativa de estos elementos que diversos autores (Fontanella de Weinberg, 1987: 110; Donni de Mirande, 2004: 127; Ramírez Luengo, 2005b: 31, 2006: 41) han apuntado en diferentes aproximaciones diacrónicas al tema, algo que quizá se deba poner en relación, siquiera como hipótesis, con esa sobreabundancia de diminutivos que caracteriza a día de hoy al español andino en general y del occidente de Bolivia en concreto (Coello Vila, 1996: 177; Mendoza Quiroga, 2008: 229).

Más allá de esta primera cuestión, lo que resulta evidente a partir de los datos del corpus es que para estos momentos se ha producido ya la decantación de los diferentes sufijos -propia de los siglos XVI y XVII, tanto en España como en América (Fontanella de Weinberg, 1992: 95-6; Ramírez Luengo, 2007: 70-1)- y la selección y generalización de -ito como único morfema diminutivo productivo: en efecto, cabe indicar que, en la totalidad de los casos de derivación apreciativa que ofrece el corpus, el sufijo empleado es el ya mencionado -ito, con ejemplos como solito (p. 14), rinconcito (p. 157), mocitos (p. 236), arribita (pp. 153, 250), Tomasito (p. 335) o armaditos (p. 342) 12; por supuesto, esta situación que ofrece el corpus en ningún caso puede sorprender si se tiene en cuenta que el proceso de cambio en cuanto al uso del diminutivo inmediatamente descrito parece producirse en la práctica totalidad de la América hispana a lo largo del siglo XVIII (Náñez Fernández, 1973: 385; Ramírez Luengo, 2006: 40), de manera que los datos obtenidos del Tambor Vargas simplemente reflejan la situación moderna mantenida hasta el día de hoy en el occidente boliviano (Coello Vila, 1996: 177) y, de paso, que también el español de esta zona ha experimentado este cambio general en momentos coincidentes con otras zonas del continente ${ }^{13}$.

11. Esto demuestra la importancia de contar con corpus de control de características homogéneas y zonas geográficas distintas en los estudios que, como este, se enclavan en la perspectiva de la dialectología histórica; sobre esta cuestión de primera importancia, resulta especialmente revelador el trabajo de Calderón (2014).

12. Todos los ejemplos se citan según la transcripción del texto que aparece en Vargas (2008); se coloca entre paréntesis, por tanto, la página de este volumen en que se descubre el ejemplo en cuestión.

13. Lo que no significa, con todo, que en la totalidad del continente el proceso de cambio se produzca exactamente al mismo tiempo, como bien demuestra Fontanella de Weinberg (1992: 96) al describir la cronología del fenómeno en diferentes variedades regionales. A este respecto, cabe señalar que una pequeña cala en el dieciochesco Diario de Sebastián de Segurola (La Paz, 1781) -equivalente desde el punto de vista geográfico y tipológico, por tanto, al corpus estudiado en estas líneas- permite mantener la idea de la vitalidad de -illo en la región hasta finales del Siglo Ilustrado, al registrar algunos ejemplos de este sufijo con pleno valor diminutivo: "se estan vendiendo dos tajadillas de cueros cocidos (..); tambien un retacillo de papel untado con dulce; pesado en dos granos de maiz"; "encontraron lagos de sangre derramada y armas que habian dejado garrotes, hondas y barretillas para cavar los paredones” (Mendoza Quiroga, 2001; doc. 85). 
En relación con lo anterior, se debe señalar también que el corpus presenta algunos ejemplos formales del sufijo -illo, pero siempre en elementos lexicalizados al estilo de hablillas (p. 18), pandilla (p. 109), paletilla (p. 243) o el topónimo Condorillo (pp. 289, 339) ${ }^{14}$, mientras que otros sufijos como -ico o -uelo son totalmente desconocidos en el texto del soldado-historiador. Se puede decir, por tanto, que, a la luz de estos datos, en la primera mitad del siglo XIX existe solo un diminutivo que funge como tal en el español boliviano occidental, -ito, y que se acompaña de determinadas voces en las que -como testigos o remanentes de una situación de variación morfológica previa- pervive un sufijo -illo lexicalizado, en una descripción que, con escasas diferencias de detalle, es aplicable a la práctica totalidad de la América hispana del momento (Ramírez Luengo, 2007: 70-1).

Dentro aún de lo estrictamente morfológico, es necesario indicar también la (prácticamente) total inexistencia del interfijo - ec- en aquellos elementos que en general lo exigen, como son las bases monosilábicas terminadas en consonante, o las bases disilábicas con diptongo en la sílaba final (Felíu, 2009: 66): en efecto, con la excepción de indiecito (pp. 191, 319), perteneciente al segundo grupo y que en la totalidad de las ocasiones ofrece el elemento -ec-, las voces que aparecen en el corpus y se engloban en los dos apartados anteriores carecen de tal elemento, con ejemplos como viejito/a (pp. 25, 256), pueblito (p. 230) y huesito (p. 295) ${ }^{15}$. Una vez más, tal situación no debe sorprender, pues ya señala Felíu (2009: 66) que “esta distribución de los interfijos está sujeta a variación dialectal, de forma que en Hispanoamérica son frecuentes diminutivos como viejito o solcito" 16 , pero es importante mencionarla porque constituye una prueba de que las tendencias descritas para el momento actual ya se han generalizado en la primera mitad del siglo XIX o, si se quiere, favorece la hipótesis de que -dentro de los procesos de dialectalización del español americano- las

14. La presencia del topónimo Condorillo no resulta extraña, pues, según la NGLE (2009: 631), en América “constituyen topónimos muchos diminutivos formados en -illo / -illa”, a lo que la obra académica añade que en este continente "son raros los formados con -ito / -ita, pero también se documentan”, algo que corrobora, en el caso concreto de este corpus, el ejemplo de Llavecita: “Mandó al capitán don Pascual García al lado de Carguani y Llavesita donde havía hecho ocultar sesenta fuciles” (p. 173).

15. Cabe indicar, además, la presencia de la voz matorralito (p. 156), que constituye un ejemplo de la tendencia -frecuente, según la NGLE (2009: 650), “en Chile y algunos países andinos”- a emplear el alomorfo -ito con "algunos sustantivos y adjetivos agudos terminados en -l”.

16. Si bien esta afirmación es en general correcta, lo cierto es que la situación es un poco más compleja, no solo por "la existencia de numerosas excepciones, tanto en el español americano como en el europeo” (NGLE, 2009: 644), sino también por el descubrimiento de diferencias de uso dentro de la propia Hispanoamérica (NGLE, 2009: 644). 
diferencias normativas registradas hoy en este punto entre el Nuevo Mundo y España existen por lo menos desde la primera mitad del Ochocientos ${ }^{17}$.

Desde otro punto de vista, resulta también de primera importancia analizar los valores semánticos que presentan los diminutivos que Vargas utiliza en su texto, así como la frecuencia de uso de tales valores significativos en el corpus, pues ambas cuestiones aportan datos relevantes a la hora de comprender en profundidad el empleo de estos elementos en el español decimonónico de la Bolivia andina. De este modo, se debe indicar en primer lugar que, como bien indica Company (2007: 109), “el diminutivo es una forma altamente polisémica que puede expresar tanto un valor referencial: la disminución del tamaño de la base, cuanto significados pragmáticos valorativos de distinta índole”18, entre los que cita "la proximidad afectiva, la ironía, el respeto, la humildad, el desprecio o la conmiseración”19; por supuesto, ambas significaciones se emplean en todas las variedades del español, pero lo que resulta interesante a este respecto es que los porcentajes de uso de ambas posibilidades crean una sutil diferenciación dialectal que tiene que ver con el predominio de uno u otro de los significados, de manera que:

el español de México suele emplear el diminutivo no por razones de semántica referencial externa, esto es, no suele usarse el diminutivo para indicar el menor tamaño de la entidad base, sino para significar diversas valoraciones de tipo pragmático que el hablante proyecta sobre esas entidades en una determinada situación comunicativa, y, por el contrario, en el español castellano no predominan los significados pragmáticos en el empleo de un diminutivo, ya que en esta variedad [...] el valor referencial de disminución del tamaño del referente compite, y predomina ligeramente, con los valores no referenciales o pragmáticos (Company, 2007: 112-3).

17. Queda por estudiar, con todo, no solo el momento en que tales preferencias se establecen en las diferentes variedades dialectales, sino también cuál de las dos, América o España, modifica la situación previa para diferenciarse de la otra, es decir, si la situación americana era la general en los Siglos de Oro y España la modificó por la incorporación del interfijo ya citado o, por el contrario, es América quien lleva a cabo en algún momento la pérdida de este elemento y se distingue, así, de lo que era la situación común anterior; aunque la falta de estudios al respecto impide responder por el momento a esta cuestión, a la vista de lo que sucede en otros muchos puntos del sistema lingüístico del español, el autor de estas líneas se inclina por la primera de las opciones.

18. La existencia de valores significativos de muy distinto tipo en estos elementos no es descubrimiento nuevo, sino que se puede retrotraer hasta el clásico artículo de Alonso (1996 [1935]), en el que el maestro navarro señala la convivencia de usos de aminoración con otros relacionados con lo emocional, que considera más abundantes (Alonso, 1996 [1935]: 19); este trabajo da lugar posteriormente a una interesante polémica cuyo resumen se expone en Martín Zorraquino (2012: 558-9).

19. Para un análisis de estos usos pragmáticos y de los valores subjetivos que conllevan en diversos dialectos del español actual -entre ellos, el de la Bolivia andina-, véase Reynoso Noverón (2005). 
Teniendo en cuenta, por tanto, las diferencias apuntadas, resulta interesante analizar en qué situación se encuentra el español de la Bolivia occidental de la primera mitad del siglo XIX, es decir, si coincide en sus usos con los del español peninsular o, por el contrario, se acerca más al estado de cosas que describe Company (2007: 112) para México y Mendoza Quiroga (2008: 229) para el español andino boliviano actual. Partiendo, así, de la descripción semántica anteriormente facilitada, es necesario comenzar diciendo que el solapamiento que en ocasiones se produce entre ambas significaciones determina la existencia de unos casos fronterizos en los que no es fácil establecer el predominio de valores referenciales o pragmáticos, según se puede apreciar en los ejemplos siguientes (ejemplos 1-3) ${ }^{20}$ :

(1) Ese día lo manda llamar a un moso llamado don Antonio Olmedo (...), y manda al pueblo de Cavari ande España a que se presente con una carguita de leña y observe los movimientos del enemigo, como bombero (p. 119).

(2) Lo mataron a palos y a lansasos, escapando el subdelegado [por la Patria] don José Manuel Arana por haver estado en otra parte, y cayó preso solamente un sobrino que tenía, don Calisto Zarabia, jovencito (p. 169).

(3) Llegan al patíbulo, lo cientan y los afucilan, todavía el pan en la voca del yndiecito no havía acabado de tragar siquiera (p. 319).

Con todo -y sin olvidar, por supuesto, la valoración discutible que pueden tener ejemplos como los anteriores-, la clasificación de los diminutivos que presenta el corpus según los valores semánticos que ofrecen en el texto es la que queda consignada en la siguiente tabla (tabla 1):

Tabla 1. Distribución semántica de los diminutivos en el corpus

\begin{tabular}{|c|c|c|}
\hline VALORES & CASOS & PORCENTAJE \\
\hline Referenciales & 61 & $42{ }^{\prime} 06 \%$ \\
\hline Pragmáticos & 84 & $57{ }^{\prime} 93 \%$ \\
\hline TOTAL & 145 & $100,00 \%$ \\
\hline
\end{tabular}

20. Tal ambigüedad significativa resulta muy habitual en el empleo del diminutivo, según pone de relieve Martín Zorraquino (2012: 558) al indicar que este elemento gramatical "en el habla, puede proyectar el abanico de sentidos mencionados más arriba sin que quepa deslindar siempre, de forma clara, el actualizado en cada caso concreto". 
Salta a la vista, por tanto, que -como no podía ser de otra forma- ambos significados conviven en el corpus que se está analizando en estas líneas, pero también resulta evidente que existe ya en estos momentos un claro predominio de los usos de tipo pragmático (ejemplos 4 y 5), que aventajan a los referenciales (ejemplos 6 y 7) en algo más de un 15\%:

(4) Entonces picó su caballo (...) y asércase más a la tropa preguntando el quién vive, los otros no contestan más que la Patria, se asercaban agachaditos por entro de los matorrales (p. 116).

(5) El desgraciado Ayllón a las 10 del día le da una puñalada a una muger llamada Manuela Navarro (alias la Gordita) en la pierna (p. 371)21.

(6) Entonses trastornando yo una lomita me eché al suelo, después de quatro pies me encajé a una quebradita muy ridícula, como abía algunos matorrales me perdí (p. 148).

(7) Entonses se iban asercando los soldados de la partida y como el ladrido de los perros ya se oíyan del pueblito de Tirco no hicieron más que picar el cura y compañeros (p. 230).

Más allá de los datos cuantitativos en sí, este hecho resulta interesante por dos cuestiones claramente relacionadas: por un lado, porque parece demostrar que para la primera mitad del siglo XIX existe ya en el uso del diminutivo del español andino boliviano una situación relativamente semejante a la que Mendoza Quiroga (2008: 229) describe para la sincronía actual; por otro -y como consecuencia de lo anterior-, porque este dato apoya la hipótesis de que los procesos de dialectalización -en este caso, en un fenómeno tan identificador de la variedad andina como el uso del diminutivo- "tienen lugar durante la época colonial, dado que en los primeros años del siglo XIX, durante las Independencias, la dialectalización del continente ya se ha consumado" (Ramírez Luengo, 2011: 18).

En relación también con este predominio de los usos pragmáticos ya mencionado, es interesante señalar que el diminutivo no aparece en el corpus únicamente junto a bases como el sustantivo o el adjetivo, sino que se descubre también con otras quizá no tan frecuentes en el español general: en efecto, si bien es verdad que el sustantivo y el adjetivo representan la mayoría de las bases a las que se añaden los sufijos apreciativos del corpus -en concreto el 75'86\% del total, es decir, 110

21. Aunque no constituye el objetivo de este trabajo, no se puede dejar de señalar a la luz de este ejemplo concreto la estrecha relación que existe entre el diminutivo y la cortesía verbal, y más en concreto su empleo en ocasiones para "amortiguar el efecto negativo de una información no deseada" (Martín Zorraquino, 2012: 561); véase este trabajo para un estudio minucioso de esta cuestión, tanto desde el punto de vista teórico como en su aplicación práctica a un texto literario del siglo XIX. 
casos del total de 145-, lo cierto es que estos aparecen también en un porcentaje nada desdeñable, de casi un 25\%, con elementos como el cuantificador todo (ejemplos 8 y 9), el adjetivo adverbializado claro (ejemplo 10) o diferentes adverbios, algunos de uso general en el español de ambos lados del Atlántico (ejemplos 11 y 12), pero otros propios de las variedades americanas de esta lengua, como ahorita -con entrada propia hasta hace muy poco en el DRAE (1992: s.v. ahorita) y significado de 'ahora mismo, muy recientemente'22 - y lueguito, entre otros (ejemplos 13, 14, 15, 16) ${ }^{23}$.

(8) Ya no abansaron, se reunieron toditos, descansaron un buen rato, se dirijieron para Quillacollo (p. 207).

(9) A las 10 del día 6 empesaron a quemar toditas las casas, maisales, trigales, algunos trillados (p. 361).

(10) Todas las voces de los oficiales del enemigo y exortaciones se oíya y aun los cintarasos con que contenían a los soldados, clarito (p. 186).

(11) Le da el tiro al comandante Fajardo cerquita a quemarropa (p. 267).

(12) Un solo yndio le grita (...) que saliesen prontito de ese abujero (p. 358).

(13) Muy cerca de dos caminos de forma que el uno ba arribita del Chume y el otro un poco más abajo (p. 392).

(14) Al capitán Moreno Lira lo destinó a retaguardia sosteniendo la carga al enemigo con quinse hombres de caballería, lueguito se bajaron estos y Moreno por últimas (p. 156).

(15) Hiciera con vos un atentado, pero no, porque sois un imfeliz soldado, y os encargo el amor a la Patria (...), que haorita en este momento desapareserá mi existencia después de padeser tantos años (p. 410).

(16) Se paró de la puerta aentrito un rato, puesto el dedo de la mano derecha a la boca (p. 410).

Así pues, también en este punto -es decir, en lo que tiene que ver con la ampliación de las categorías a las que se añade el diminutivo-, la situación que refleja este

22. Cabe mencionar, por cierto, que estos ejemplos de ahorita parecen ser, si no lo más antiguos registrados hasta el momento, sin duda de los primeros que se tiene constancia, habida cuenta de que los casos más tempranos que ofrece CORDE [Consulta: 21/03/2014] se fechan a finales del siglo XIX, en concreto en 1870 con varios ejemplos del argentino Mansilla.

23. De hecho, se puede decir que es precisamente el uso frecuente del diminutivo con estos elementos el que determina la preponderancia de los usos denominados pragmáticos en el corpus, pues, como señala Company (2007: 49), “desde un punto de vista estricto de semántica denotativa, el tamaño de la base de esos referentes (...) no puede ser objetivamente disminuido”. Si se tiene en cuenta exclusivamente los adjetivos y sustantivos del corpus -esto es, aquellos elementos en los que el hablante puede añadir el sufijo diminutivo para expresar cualquiera de los dos valores semánticos señalados-, la situación porcentual sufre un cambio de cierta importancia: así, en este caso se registran 61 ejemplos de valor referencial frente a 49 de carácter pragmático, esto es, un 55’45\% de los primeros y un 44’54\% de los segundos, que resultan ahora más escasos cuantitativamente. 
corpus decimonónico se asemeja a la que actualmente presenta esta misma zona (Mendoza Quiroga, 2008: 229), si bien se debe precisar que no resulta exactamente igual, habida cuenta de que el Diario no ofrece ningún caso de sufijo apreciativo con elementos como pronombres, numerales, posesivos o deícticos, frecuentes hoy en la zona andina (NGLE, 2009: 634, 1398); se hace necesario interrogarse, por tanto, si tal ausencia se debe a que todavía no se dan en el español de la época casos como suyito, ahicito y dositas -lo que implicaría una generalización posterior del diminutivo a bases como estas- o a que, aun dándose en la oralidad de -al menos- algunos sociolectos del español andino boliviano del momento, no hacen su aparición en el texto por motivaciones difíciles de precisar y que quizá se puedan relacionar con lo diastrático, lo diafásico o incluso lo tipológico, entendiendo como tal las convenciones literarias a las que se sujeta un texto del estilo del aquí analizado.

4. De este modo, el análisis llevado a cabo hasta el momento sobre el Diario del Tambor Vargas permite extraer una serie de conclusiones de notable interés acerca de la situación que presenta el diminutivo en la Bolivia andina de la primera mitad del siglo XIX, así como de su empleo en esta variedad del español; en concreto, tales conclusiones tienen que ver con la morfología de estos elementos, con sus valores semánticos y con las bases a las que se añaden, pero en realidad se relacionan con una cuestión mucho más general, a saber, si el estado de cosas que hoy se describe respecto a estos elementos existe ya en la primera parte del Ochocientos o es, por el contrario, un proceso que tiene lugar durante sincronías posteriores, más cercanas a la actual.

Así pues, desde un punto de vista estrictamente morfológico de estos elementos, cabe señalar que se descubre una situación muy semejante a la actual, tanto en lo que tiene que ver con el predominio absoluto de -ito como único sufijo productivo -acompañado, eso sí, de casos lexicalizados de -illo que se entienden desde la convivencia de diminutivos propia de los siglos XVI y XVII (Fontanella de Weinberg, 1992: 95-6; Ramírez Luengo, 2007: 70-1)-, como en lo que se refiere a la morfología de este elemento, que muestra ya la ausencia del interfijo - ec- en los contextos donde este se emplea en España, lo que resulta característico actualmente (de muchas variedades) del español americano (Felíu, 2009: 66)²4.

24. Dada la falta de estudios históricos acerca del uso de este interfijo en los diminutivos, es imposible concluir por el momento si se debe decir que "muestra ya la ausencia" o, por el contrario, que "muestra todavía la ausencia”; sin duda constituye este uno de los temas que todavía hay que abordar para trazar una correcta historia de estos sufijos. 
En cuanto a las significaciones que posee el diminutivo, el análisis del corpus también descubre una situación cercana a la actual, con la convivencia -como no podía ser menos- de usos pragmáticos y referenciales, pero con cierta tendencia al predominio de los primeros que se puede relacionar con ese "sentido afectivo" que, según Mendoza Quiroga (2008: 229), es especialmente frecuente en el español de la Bolivia andina actual ${ }^{25}$; desgraciadamente, la ausencia -o el desconocimiento del autor de estas líneas- de datos porcentuales acerca de esta cuestión en el español actual de la zona impide comprobar si tal tendencia a privilegiar estos usos frente a los meramente referenciales se ha mantenido constante entre el siglo XIX y el momento actual o, por el contrario, se ha experimentado una ampliación semejante, por ejemplo, a la que Company (2007: 49) apunta para el español mexicano durante la época colonial.

En relación con lo anterior, también coinciden los usos que refleja Vargas con la situación actual en lo que tiene que ver con la ampliación de bases léxicas a las que se añaden estos elementos, que no se circunscriben a sustantivos, adjetivos o determinados cuantificadores, sino que se extienden también a adverbios como ahora, luego o adentro; es necesario indicar, con todo, que la situación no es exactamente igual, pues en el corpus no se registra ningún ejemplo de sufijación apreciativa con categorías como los pronombres, los posesivos o los numerales, fenómeno muy identificador del español andino y que, de acuerdo con los datos que ofrece este corpus, resulta aún desconocido en el periodo 1800-1850.

Por supuesto, el presente trabajo -por más que ofrezca cierta luz sobre el empleo de estos elementos en el Ochocientos boliviano- deja aún muchas cuestiones por responder, e incluso abre nuevas preguntas que deberán ser contestadas en el futuro: dada la ya descrita ampliación de bases que se descubre en este momento, por ejemplo, se hace ineludible analizar en qué momento -y por qué razones- tiene lugar este fenómeno que, en el caso concreto de México, Company (2007: 49) registra ya para el siglo XVIII26; en esta línea, es así mismo

25. De hecho, este autor (Mendoza Quiroga, 2008: 229) indica que tal estado de cosas es especialmente frecuente en el caso de los hablantes bilingües, grupo en el que, como se dijo ya, cabe incluir al informante que constituye la base de este estudio.

26. Cabe señalar al respecto que una búsqueda minuciosa en los documentos dieciochescos del Alto Perú que edita Mendoza Quiroga (2001) no ofrece ni un solo ejemplo de diminutivo con otras categorías léxicas que no sean sustantivos o adjetivos; si bien se puede considerar que la tipología mayoritaria en el corpus -documentación notarial de mayor o menor formalidad- puede determinar la ausencia de ejemplos como ahorita o lueguito, lo cierto es que también aparecen en él otros textos como cartas privadas (documento 74) -a veces incluso de bilingües (documento 83) - o el interesantísimo Diario de Segurola (La Paz, 1781) (documento 85), que en ningún caso se pueden considerar documentos más formales o de registro más elevado que el escrito de Vargas. 
necesario investigar - como ya se dijo con anterioridad- si la ausencia de bases sufijadas con diminutivo como pronombres, posesivos y numerales constituye un fenómeno del español de la época y, por tanto, una diferencia entre la sincronía analizada y la actual, o simplemente responde a circunstancias propias del corpus seleccionado para este trabajo, es decir, si se trata de una cuestión de carácter lingüístico o, por el contrario, de un hecho puramente textual, relacionado con las fuentes de estudio.

Por último, el hecho de que tal estado de cosas se registre en un hablante bilingüe -o mejor, trilingüe- demuestra, obviamente, que el uso del diminutivo que hoy resulta propio del español de los Andes bolivianos se daba ya durante el siglo XIX en hablantes con tal característica, pero en ningún caso evidencia que, como aventuran algunos estudiosos (Caravedo, 1992: 295; Godenzzi, 1996: 90; Mendoza Quiroga, 2008: 229), el contacto lingüístico sea la causa que determina el origen del fenómeno; se hace preciso ahora, por tanto, estudiar los usos del diminutivo en hablantes bolivianos decimonónicos monolingües, para ver de este modo si el empleo de estos elementos es semejante en ambos grupos o, por el contrario, existen diferencias que se pueden achacar al influjo de las lenguas autóctonas sobre el sistema del español.

De este modo, se pueden concluir estas líneas diciendo que la situación que ofrece el diminutivo en la Bolivia andina de la primera mitad del siglo XIX -tal y como la refleja el Diario de José Santos Vargas- resulta muy semejante a la que se ha descrito para la sincronía actual de la región en cuestiones como el empleo de -ito como sufijo productivo único, diversas cuestiones relacionadas con la morfología de este elemento -en concreto, con la distribución de los alomorfos -ito y -ecito-, la clara preferencia por los empleos pragmáticos frente a los referenciales y, como consecuencia de lo anterior, la extensión de la derivación apreciativa a algunas bases - no todas- que hoy la aceptan, como son los adverbios de lugar y de tiempo (arribita, adentrito, lueguito o ahorita); es posible sostener, por tanto, que un rasgo idiosincrásico y fuertemente identificador del español boliviano occidental de hoy existe en la zona por lo menos desde mediados del siglo XIX, lo que implica que en este punto concreto del sistema -como en otros- la dialectalización se ha producido ya para estos momentos y que, en el caso concreto del Tambor Vargas, resulta más que probable que su forma de hablar, en lo que a los diminutivos se refiere, no se diferenciase mucho de lo que hoy se puede escuchar en La Paz, en Potosí o en cualquier otro lugar de los Andes bolivianos. 


\section{Bibliografía}

ALONSO, A. (1996 [1935]). Noción, emoción, acción y fantasía en los diminutivos. Madrid: Gredos.

ARNADE, Ch. (1972). La dramática insurgencia de Bolivia. La Paz: Juventud.

CALDERÓN, M. (2014). "Muestras de oralidad en el Corpus Diacrónico del Español del Reino de Granada (siglo XVIII)”. Oralia 17: 117-145.

CARAVEDO, R. (1992). "El atlas lingüístico hispanoamericano en el Perú: observaciones preliminares”. Lingüística Española Actual 14: 287-299.

COELLO VILA, C. (1996). "Bolivia”, en Manual de dialectología hispánica. El español de América (Dir. M. Alvar). Barcelona: Ariel, 169-183.

COMPANY, C. (2007). El siglo XVIII y la identidad lingüística de México. México DF: UNAM; Academia Mexicana de la Lengua.

DEMÉLAS, M. D. (2007). Nacimiento de la guerra de guerrillas. El Diario de José Santos Vargas (1814-1825). La Paz: IFEA; Plural.

DONNI DE MIRANDE, N. E. (2004). Historia del español en Santa Fe del siglo $X V I$ al siglo XIX. Buenos Aires: Academia Argentina de Letras.

FELÍU, E. (2009). "Palabras con estructura interna”, en Panorama de la lexicología (Ed. E. de Miguel). Barcelona: Ariel, 51-82.

FONTANELLA DEL WIENBERG, M. ${ }^{a}$ B. (1987). El español bonaerense. Cuatro siglos de evolución lingüistica (1580-1980). Buenos Aires: Hachette.

FONTANELLA DE WEINBERG, M. ${ }^{a}$ B. (1992). El español de América. Madrid: MAPFRE.

FRAGO GRACIA, J. A. (2011). "El español de América en la Independencia. Adiciones gramaticales, I”. Boletín de Filología de la Universidad de Chile 46 (1): 47-74.

GODENZZI, J. C. (1996). "Transferencias lingüísticas entre el quechua y el español”. Signo y Seña 6: 73-99.

GÓMEZ GONZÁLEZ, A. (2013). El léxico boliviano del siglo XIX: análisis del Diario del Tambor Vargas (Trabajo de Fin de Grado inédito). Alcalá de Henares: Universidad de Alcalá.

GÓMEZ SEIBANE, S. (2012). "Neutralización de género, omisión y duplicaciones de objetos en el español boliviano de la Independencia”, en Por sendas ignoradas. Estudios sobre el español del siglo XIX (Ed. J. L. Ramírez Luengo). Lugo: Axac: 75-97.

LÁZARO MORA, F. (1999). "La derivación apreciativa”, en Gramática descriptiva de la lengua española (coords. I. Bosque y V. Demonte). Madrid: Espasa-Calpe, III: 4645-4682. 
MACHICADO, C. et al. (2009). "Elecciones en la guerrilla de Ayopaya según el diario del Tambor Vargas (1814-1824)”. Revista Ciencia y Cultura 10: 45-104.

MAMANI SIÑANI, R. L. (2010). "La División de los Valles”: estructura militar, social y étnica de la guerrilla de La Paz y Cochabamba (1814-1817). La Paz: Instituto de Estudios Bolivianos; ASDI; Sarec.

MARQUILHAS, R. (2000). A Faculdade las Letras. Leitura e escrita em Portugal do século XVII. Lisboa: Imprensa Nacional-Casa da Moeda.

MARTÍN ZORRAQUINO, M. A. (2012): "Sobre los diminutivos en español y su función en una teoría de la cortesía verbal (con referencia especial a un cuento de Antonio de Trueba", en Cum corde et in nova grammatica: estudios ofrecidos a Guillermo Rojo (coords. T. E. Jiménez Juliá, B. López Meirama, V. Vázquez Rozas, A. Veiga Rodríguez). Santiago de Compostela: Universidade de Santiago de Compostela: 555-569.

MENDOZA, G. (2008). "Introducción”, en Diario de un combatiente de la Guerra de Independencia (1814-1825). La Paz: ABNB; Plural: XXIII-LXV. MENDOZA QUIROGA, J. G. (2001). "III. Bolivia: a) Alto Perú. Siglos XVIXVIII”, en Documentos para la Historia Lingüística de Hispanoamérica, III (Ed. E. Rojas). Tucumán: Universidad Nacional de Tucumán.

MENDOZA QUIROGA, J. G. (2008). "Bolivia”, en El español en América. Contactos lingüísticos en Hispanoamérica (coord. A. Palacios). Barcelona: Ariel: 213-236.

NÁÑEZ FERNÁNDEZ, E. (1973). El diminutivo. Historia y funciones en el español clásico y moderno. Madrid: Gredos.

RAMÍREZ LUENGO, J. L. (2005). "Notas sobre el uso del diminutivo en Guipúzcoa y Navarra en el siglo XVIII”, en Actas del III Congreso Nacional de la Asociación de Jóvenes Investigadores de Historiografía e Historia de la Lengua Española (coord. C. Cazorla et al.). Jaén: Universidad de Jaén: 399-408.

RAMÍREZ LUENGO, J. L. (2005b). "Variación gramatical y tipos textuales: el diminutivo en la época de Don Quijote". Ámbitos 13: 29-34.

RAMÍREZ LUENGO, J. L. (2006). "Una nota de sociolingüística histórica: el diminutivo en el español uruguayo del siglo XIX”. Res Diachronicae 5: 39-45.

RAMíREZ LUENGO, J. L. (2007). Breve historia del español de América. Madrid: ArcoLibros.

RAMíREZ LUENGO, J. L. (2011). La lengua que hablaban los próceres. El español de América en la época de las Independencias. Buenos Aires: Voces del Sur. 
REAL ACADEMIA ESPAÑOLA (1992). Diccionario de la lengua española (21 ${ }^{\mathrm{a}}$ ed.). Madrid: Espasa-Calpe.

REAL ACADEMIA ESPAÑOLA (2014). Diccionario de la lengua española (23 ed.). Madrid: Espasa-Calpe.

REAL ACADEMIA ESPAÑOLA y ASOCIACIÓN DE ACADEMIAS DE LA LENGUA ESPAÑOLA (2009). Nueva gramática de la lengua española. Madrid: Espasa-Calpe.

REYNOSO NOVERÓN, J. (2001-2002). "Desarrollos paralelos en el contacto español-lenguas indígenas: indigenismos léxicos y diminutivos”. Anuario de Lingüística Hispánica 17-18: 111-128.

REYNOSO NOVERÓN, J. (2005). "Procesos de gramaticalización por subjetivización: el uso del diminutivo en el español”, en Selected Proceedings of the $7^{\text {th }}$ Hispanic Linguistic Symposium (ed. D. Eddington). Somerville: Cascadilla Proceedings Project: 79-86.

VARGAS, J. S. (2008). Diario de un combatiente de la Guerra de la Independencia (1814-1825). La Paz: ABNB; Plural. 